\title{
True, false, paranormal and 'designated'?: a reply to Jenkins*
}

\author{
COLIN CARET \& AARON J. COTNOIR
}

\section{- Introduction}

Jenkins [4] charges that the language advanced in Beall [2] is either expressively impoverished, or inconsistent. We argue that Jenkins' objections are based on unreasonably strong constraints on formal theories of truth. Our primary concern is not to defend the 'paranormal' framework advanced in Beall [2], but to respond to a common - and implausible - 'revenge'-style charge directed at a certain class of formal theories of truth and paradox. In $\$ 1$, we outline Beall's language; $\$ 2$ outlines Jenkins' main criticism and the resulting dilemma. In $\$ 3$, we give our two-fold reply to Jenkins.

\section{True, false and paranormal}

In Beall [2] the following many-valued language $(\mathscr{L})$ is defined.

The semantic values are $V=\{1,0.75,0.5,0.25,0\}$

The designated values are $D=\{1,0.75\}$

The connectives and predicates are defined in the following way:

$$
\begin{gathered}
\nu(A \& B)=\min \{\nu(A), \nu(B)\} \\
\nu(A \vee B)=\max \{\nu(A), \nu(B)\} \\
\nu(\neg A)=1-\nu(A) \\
\nu(T\langle A\rangle)=\nu(A) \\
\nu(F\langle A\rangle)=\nu(T\langle\neg A\rangle) \\
\nu(\pi A)=\left\{\begin{array}{l}
0 \text { if } \nu(A) \in\{1,0\} \\
\text { otherwise } 0.75
\end{array}\right.
\end{gathered}
$$

The intended interpretation is that the predicates $T$ and $F$ represent 'true' and 'false' in the object language. These predicates satisfy the fundamental intersubstitutability of $T\langle A\rangle$ with $A$, and of $F\langle A\rangle$ with $\neg A$, for all sentences $A$ in all non-opaque contexts. The connective $\pi$ represents the semantic category 'paranormal' which is meant as

*forthcoming in Analysis 68.3, July 2008. (C) Colin Caret and Aaron Cotnoir. 
a colorless term for capturing all problematic, paradoxical sentences that defy easy characterization in terms of truth and falsity. Valid inferences for such a language are those which are designation-preserving.

Given a standard treatment of predicate extensions, the extension of $T$ in any model of $\mathscr{L}$ is $T^{+}=\{x: 0.75 \leq v(x)\} .{ }^{1}$ As can easily be checked, this set is negationconsistent, hence it meets the goal of 'non-triviality' defined below as NTP. Moreover, for every sentence $A$, in any model of $\mathscr{L}$, there is a designated sentence $\varphi A$ where $\varphi$ is one of $T$ or $F$ or $\pi$. This demonstrates that $\mathscr{L}$ meets the goal of 'exhaustive characterization' defined below as ECP.

(NTP) explain how, despite having both a truth predicate (in our language, for our language) and Liar sentences, our language is non-trivial.

(ECP) explain how, if at all, we can truly characterize - specify the 'semantic status of' - all sentences of our language (in our language).

These desiderata are achieved by employing overlapping semantic categories, an idea sometimes advanced by dialetheists as a reason to accept the existence of true contradictions, i.e. truths of the form $A \& \neg A$. Since the extension of $T$ in $\mathscr{L}$ is negationconsistent, it follows that there are no true contradictions. Hence, $\mathscr{L}$ provides a counterexample to the inference from satisfaction of NTP and ECP by overlap to the conclusion of dialetheism.

\section{Jenkins' argument}

In Jenkins [4] it is objected that the following predicate cannot be expressed in Beall's language.

$$
\nu(\mathrm{D}\langle A\rangle)=\left\{\begin{array}{l}
1 \text { or } 0.75 \text { if } \nu(A) \in D \\
0.25 \text { or } 0 \text { if } \nu(A) \notin D
\end{array}\right.
$$

The intended interpretation is that the predicate $\mathrm{D}$ represents the notion of designation used in the semantic machinery of the meta-theory. This predicate cannot be expressed because of the following sentence, $A^{*}$.

$\left(A^{*}\right) \neg \mathrm{D}\left\langle A^{*}\right\rangle$

If $\nu\left(A^{*}\right) \in D$, then $\nu\left(\mathrm{D}\left\langle A^{*}\right\rangle\right) \in D$, but then $\nu\left(\neg \mathrm{D}\left\langle A^{*}\right\rangle\right) \in\{0,0.25\}$ so by the definition of $A^{*}$ it follows that $\nu\left(A^{*}\right) \notin D$. If $\nu\left(A^{*}\right) \notin D$, then $\nu\left(\mathrm{D}\left\langle A^{*}\right\rangle\right) \in\{0,0.25\}$, but then $\nu\left(\neg \mathrm{D}\left\langle A^{*}\right\rangle\right) \in D$ so by the definition of $A^{*}$ it follows that $\nu\left(A^{*}\right) \in D$. The designation predicate $\mathrm{D}$ cannot be expressed on pain of a sentence that is designated iff it is undesignated.

Jenkins calls this fact about $A^{*}$ a 'model-theoretic analogue of true contradictions' ([4], 81). From this, she draws the following dilemma for Beall.

[E]ither no model language is fully adequate as a model of English, which can talk about its own semantic machinery, or, like the model languages, English cannot express claims about its own semantic machinery.

\footnotetext{
${ }^{1}$ For convenience, we write ' $T$ ', to denote the extension of $T$.
} 
If one takes the first option, then one has to admit that (Beall's discussion of) these models can't help us defend exhaustive characterization or understand the Liar-like paradoxes. [...] Finally, of course, if one takes the second option, one places a familiar, and implausible, kind of limitation on the expressive powers of English. ([4], 83-84)

Against Jenkins, we argue that this is a false dilemma. Both horns assume that the expressibility of a predicate like $\mathrm{D}$ is essential to adequately model the semantics of English; that any model language which fails to do so fails to satisfy ECP. But this is an assumption that one might reject. ${ }^{2}$ On the intended reading of 'exhaustive', an exhaustive characterization would simply require that for every sentence there is at least one semantic predicate we can use to (truly) characterize it. As we previously noted, Beall's language features enough predicates to achieve this goal.

\section{Reply}

Jenkins appears to be assuming a much more robust 'exhaustive' characterization principle when she writes,

[T] he ability of a language to express claims about its own semantic machinery is what the exhaustive characterization project is supposed to secure $[\ldots]$ The kind of exhaustive characterization we want, and which Priest thinks leads to acceptance of true contradictions, is an exhaustive characterization of all the sentences in the model in terms of their semantic values. $([4], 82)$

This suggests a requirement like ECP*.

$\left(\mathrm{ECP}^{*}\right)$ explain how, if at all, we can truly characterize all sentences of our language in terms that uniquely specify their treatment under the 'semantic machinery' of the proffered formal model.

This robust conception of 'exhaustive characterization' requires that formal models of our language be capable of far more than the expression of semantic predicates sufficient to categorize English sentences. It requires that such models be capable of expressing every semantic value that plays a role in the formal mechanism. This conception of 'exhaustive characterization' explains Jenkins' demand for the expressibility of a designation predicate, but Jenkins has failed to provide reasons for preferring ECP* over ECP.

We believe that Jenkins is conflating purely model-dependent, instrumental notions like 'semantic value' and 'designation' with predicates that actually play a role in the semantics of English. As such, the predicate D is not semantically significant, and need not be expressible. This gives the lie to ECP*: such a conception of the characterization project is based on a too-literal understanding of the explanatory power of the formal tools involved in modeling our language.

\footnotetext{
${ }^{2}$ Indeed, Beall [1] explicitly argues against such an assumption, as does Field [3].
} 


\subsection{Semantic values?}

First, why think the semantic values need to be expressed individually? There are five semantic values in $\mathscr{L}$ which correspond to the ways in which the three semantic categories 'true', 'false', and 'paranormal' overlap. The below diagram illustrates how Beall conceives of the relation of these semantic categories and how the semantic values fall out of these relations.

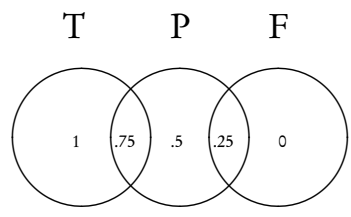

The semantic values, then, are heuristic tools used to signal a sentence's location among the overlapping semantic categories. The three categories which give rise to these five 'locations' are non-trivially expressible in the model-language. Beall can, in fact, predicate (truly) of each sentence its location among the semantic categories. ${ }^{3}$ What one cannot do in $\mathscr{L}$ is to (truly) characterize each sentence in terms of the semantic categories that don't apply, as it were. For instance, given a sentence $A$ such that $\nu(A)=0.5$ we might think of $A$ as 'not true' and 'not false', but we cannot say as much since $\nu(\neg T\langle A\rangle \& \neg F\langle A\rangle) \notin D$. If this failure of the language is what Jenkins is concerned about, it points in the direction of a very robust conception of 'exhaustive' characterization indeed.

This suggests a project which aims to (truly) characterize all sentences both exhaustively and exclusively, that is, under semantic predicates that express each semantic value to the exclusion of every other value. Why expect this? Jenkins has not argued for the desirability of 'exclusive' characterization, nor has Beall said anything to commit him to such a project. In fact, the fruitfulness of overlapping semantic categories as a tactic for understanding the non-triviality of truth seems to depend on a lack of such predicates. If Jenkins is advocating this aim, then her argument cuts equally against the semantics for Graham Priest's $L P$ and Anderson and Belnap's $F D E{ }^{4}$ Ruling out the adequacy of this entire family of semantics on the grounds of an unargued methodological stance is unreasonable.

\subsection{Designation?}

Second, why think designation is semantically significant? Jenkins suggests it is the notion of designation which is playing the semantic role that 'true' plays in English.

But ' $T$ ', in Beall's model, does not express the model-theoretic analogue of the semantic property that our English predicate 'true' expresses. In this model, the role of that property is played by designation, which, as

\footnotetext{
${ }^{3}$ As can be checked for all $A$, if $v(A)=1$, then $v(T\langle A\rangle)=1$; if $\nu(A)=0.75$, then $v(T\langle A\rangle \& \pi A)=0.75$; if $\nu(A)=0.5$, then $\nu(\pi A)=0.75$; if $\nu(A)=0.25$, then $\nu(\pi A \& F\langle A\rangle)=0.75$; if $\nu(A)=0$, then $\nu(F\langle A\rangle)=1$.

${ }^{4}$ For example, in the case of Priest's $L P$, consider the predicate $Z$, where $v(Z\langle A\rangle)=1$ if $\nu(A)=0$, and otherwise $\nu(Z\langle A\rangle)=0$. Now consider the sentence $\lambda: Z\langle\lambda\rangle$. Clearly, ECP* causes triviality for $L P$ as well.
} 
we have seen, is not definable in the language. Hence the language is not, despite appearances, semantically closed. ([4], 82)

We argue that this is mistaken. Designation in the meta-language is meant to model $T$ in the object-language. We note that the designated values, $D=\{1,0.75\}$, and the extension of 'true', $T^{+}=\{x: 0.75 \leq \nu(x)\}$, are such that for any sentence $A, \nu(A) \in D$ iff $A \in T^{+}$. That is, $A$ has a designated value exactly when it is true. So, designation is a meta-linguistic device which models the extension of 'true', and this should be enough to answer Jenkins' stated charge. ${ }^{5}$

There is, however, an implicit, stronger requirement in Jenkins' charge. In defining $\mathrm{D}$ as her desired truth predicate, Jenkins indicates that whatever plays the role of 'truth' should be such that $\mathrm{D}\langle A\rangle$ is false in cases where $A$ is undesignated. This enables us to truly say (by use of negation) that a sentence is not true. That is, she wants the behavior of 'true' to deliver a dual notion of 'untrue' that is distinct from 'false'. She seems to hold that such a feature is essential to the role of the English predicate 'true, ${ }^{6}$ but why would one require or think this?

Jenkins has provided no argument for the requirement that 'untruth' be expressible; but the requirement appears to be based on an unwarranted assumption, namely that 'true' in English is a classical notion. Jenkins seems to be exploiting the fact that our meta-language is formulated in classical set-theory, according to which, for any $x$ and set $Y$ either $x \in Y$ or $x \notin Y$. It is no wonder, then, that for any sentence $A$, either $\nu(A) \in D$ or $\nu(A) \notin D$. And it is because of this fact that ' $\mathrm{D}$ ' is not expressible. As we know, classical logic cannot satisfy both NTP and ECP (and hence ECP*) when liar sentences are included in the language, so it comes as no surprise that classically bivalent notions will yield inconsistency.

We conclude that Jenkins has provided no motivation for the semantic significance of designation or for ECP*; hence, her arguments in no way undermine Beall's principal claim. In general, the above considerations suggest that ECP* is far too unreasonable of a requirement. It appears doubtful, then, that 'revenge'-style objections based on such assumptions pose any problems for formal theories of truth which aim to solve the paradoxes. ${ }^{7}$

\footnotetext{
${ }^{5}$ Although Jenkins never mentions validity, the notion of designation most obviously plays a role in the definition of argument forms which are valid-in- $\mathscr{L}$. On any reading, validity counts as a 'semantically significant' notion of English. The question is how to understand the relation between validity and validin- $\mathscr{L}$. We deny the inference from (1) designation is used to model validity, and (2) validity is semantically significant, to the conclusion that (3) designation is semantically significant. For the above reasons, we claim that purely model-theoretic notions useful for modeling semantically significant phenomena need not themselves be semantically significant. There may be a related concern in this area about whether Beall's language can enjoy a validity relation. This hangs largely on whether he can construct a suitable conditional that avoids Curry paradoxes. Since this is not addressed in any length in Beall [2], we leave the issue aside.

${ }^{6}$ In fact, Jenkins has pointed out in private correspondence that she thinks the expression of the "untruth' of a sentence in the above sense is an important requirement. She maintains that any language which fails to meet this requirement in expressively incomplete, although has not provided any argument in support of it.

${ }^{7}$ Thanks to JC Beall and Carrie Jenkins for helpful comments on earlier drafts of this paper.
} 


\section{aaron.cotnoir@uconn.edu \\ colin.caret@uconn.edu}

\section{References}

[1] Beall, JC. 2007. Prolegomena to future revenge Revenge of the Liar. JC Beall (ed.) Oxford University Press.

[2] Beall, JC. 2006. True, false and paranormal. Analysis. 66: 102-14.

[3] Field, H. 2007. Solving the paradoxes, escaping revenge. Revenge of the Liar. JC Beall (ed.) Oxford University Press.

[4] Jenkins, C. 2007. True, false, paranormal, and designated: a reply to Beall. Analysis 67: 80-83. 